\title{
On minimal forbidden subgraphs for the class of EDM-graphs
}

\author{
Gašper Jaklič \\ FMF and IMFM, University of Ljubljana, \\ Jadranska 19, 1000 Ljubljana, Slovenia \\ and \\ IAM, University of Primorska, Slovenia \\ Jolanda Modic* \\ XLAB d.o.o., Pot za Brdom 100, 1000 Ljubljana, Slovenia \\ and \\ FMF, University of Ljubljana, Slovenia
}

Received 2 April 2013, accepted 2 April 2014, published online 21 November 2014

\begin{abstract}
In this paper, a relation between graph distance matrices and Euclidean distance matrices $(\mathrm{EDM})$ is considered. Graphs, for which the distance matrix is not an EDM (NEDMgraphs), are studied. All simple connected non-isomorphic graphs on $n \leq 8$ nodes are analysed and a characterization of the smallest NEDM-graphs, i.e., the minimal forbidden subgraphs, is given. It is proven that bipartite graphs and some subdivisions of the smallest NEDM-graphs are NEDM-graphs, too.
\end{abstract}

Keywords: Graph, Euclidean distance matrix, distance, eigenvalue.

Math. Subj. Class.: 15A18, 05C50, 05C12

\section{Introduction}

A matrix $D \in \mathbb{R}^{n \times n}$ is Euclidean distance matrix (EDM), if there exist $\boldsymbol{x}_{1}, \boldsymbol{x}_{2}, \ldots, \boldsymbol{x}_{n} \in$ $\mathbb{R}^{r}$, such that $d_{i j}=\left\|\boldsymbol{x}_{i}-\boldsymbol{x}_{j}\right\|_{2}^{2}, i, j=1,2, \ldots, n$. The minimal possible $r$ is called the embedding dimension (see [2], e.g.).

\footnotetext{
* Corresponding author. This research was funded in part by the European Union, European Social Fund, Operational Programme for Human Resources, Development for the Period 2007-2013. The author would like to thank XLAB d.o.o., Dr. Daniel Vladušič and Dr. Gregor Berginc for all the support.

E-mail addresses: gasper.jaklic@fmf.uni-lj.si (Gašper Jaklič), jolanda.modic@ gmail.com (Jolanda Modic)
} 
Euclidean distance matrices were introduced by Menger in 1928 and have received a considerable attention. They were studied by Schoenberg [13], Young and Householder [14], Gower [4], and many other authors. In recent years many new results were obtained (see $[5,7,8,11]$ and the references therein).

They are used in various applications in linear algebra, graph theory, geodesy, bioinformatics, chemistry, e.g., where frequently a question arises, what can be said about a set of points, if only interpoint distance information is known. Some examples can be found in [2].

EDMs have many interesting properties. They are symmetric, hollow (i.e., with only zeros on the diagonal) and nonnegative. The sum of their eigenvalues is zero and they have exactly one positive eigenvalue (for a nonzero matrix). Schoenberg ([13]), Hayden, Reams and Wells ([5]) gave the following characterization of EDMs.

Theorem 1.1. Let $D \in \mathbb{R}^{n \times n}$ be a nonzero symmetric hollow matrix and let $e \in \mathbb{R}^{n}$ be the vector of ones. The following propositions are equivalent:

(a) The matrix D is EDM.

(b) For all $\boldsymbol{x} \in \mathbb{R}^{n}$ such that $\boldsymbol{x}^{T} \boldsymbol{e}=0, \boldsymbol{x}^{T} D \boldsymbol{x} \leq 0$.

(c) The matrix $D$ has exactly one positive eigenvalue and there exists $\boldsymbol{w} \in \mathbb{R}^{n}$ such that

$$
D \boldsymbol{w}=\boldsymbol{e}
$$

and $\boldsymbol{w}^{T} \boldsymbol{e} \geq 0$

Throughout the paper we will use the notation $e$ for the vector of ones of appropriate size. Vectors $\boldsymbol{e}_{i}$ will denote the standard basis.

Let $\mathcal{G}$ be a graph with a vertex $\operatorname{set} \mathcal{V}(\mathcal{G})$ and an edge set $\mathcal{E}(\mathcal{G})$. Let the distance $d(u, v)$ between vertices $u, v \in \mathcal{V}(\mathcal{G})$ be defined as their graph distance, i.e., the length of the shortest path between them. Let $G:=[d(u, v)]_{u, v \in \mathcal{V}(\mathcal{G})}$ be the distance matrix of $\mathcal{G}$.

If the graph distance matrix of a graph is EDM, the graph is called an EDM-graph. Otherwise the graph is a NEDM-graph.

Graph distance matrices of EDM-graphs were studied in several papers. Path and cycles were analysed in [9]. Star graphs and their generalizations were considered in [6, 10]. Some results on Cartesian products of EDM-graphs are also known (see [11]). However, the characterization of EDM-graphs in general is still an open problem.

In this paper, all simple connected non-isomorphic graphs on $n \leq 8$ nodes are analysed and a characterization of the smallest NEDM-graphs, i.e., the minimal forbidden subgraphs, is given.

In algebraic graph theory, a lot is known on the adjacency matrix and the Laplacian matrix of a graph. Many results on their eigenvalues exist, but not much is known on the graph distance matrix. Hopefully, this paper will provide a deeper insight into the relation between general graphs or networks and EDM theory.

There are some interesting possibilities of application. Molecular conformation in bioinformatics, dimensionality reduction in statistics, 3D reconstruction in computer vision, just to name a few.

The structure of the paper is as follows. In Section 2, all NEDM-graphs on $n \leq 8$ nodes are considered. Analysis of their properties enables us to find some larger NEDM-graphs, which are presented in sections 3 and 4. A proof that bipartite graphs are NEDM-graphs 
is given. We present two families of subdivision graphs of the smallest NEDM-graphs that are NEDM-graphs, too.

There exist graphs, for which the system (1.1) has no solution. Such graphs are studied in Section 5.

The paper is concluded with an example, where we show that not all subdivisions of graphs result in NEDM-graphs.

\section{The smallest NEDM-graphs}

In this section we consider simple connected non-isomorphic graphs on $n \leq 5$ nodes and find the smallest NEDM-graphs.

There is one simple connected graph on 2 nodes, the path graph $\mathcal{P}_{2}$, and there exist only two simple connected graphs on 3 nodes, the path graph $\mathcal{P}_{3}$ and the cycle graph $\mathcal{C}_{3}$. In [9] it was proven that path graphs and cycle graphs are EDM-graphs.

For $n=4$, there are 6 simple connected graphs (see Fig. 1). First four of them are the star graph $\mathcal{S}_{4}$, the path graph $\mathcal{P}_{4}$, the cycle graph $\mathcal{C}_{4}$ and the complete graph $\mathcal{K}_{4}$, respectively, which are EDM-graphs (see [9, 10]). Therefore we only need to consider the last two graphs, $\mathcal{G}_{4}^{(5)}$ and $\mathcal{G}_{4}^{(6)}$.

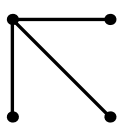

$\mathcal{G}_{4}^{(1)}$

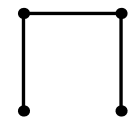

$\mathcal{G}_{4}^{(2)}$

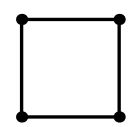

$\mathcal{G}_{4}^{(3)}$

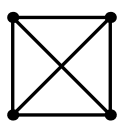

$\mathcal{G}_{4}^{(4)}$

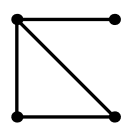

$\mathcal{G}_{4}^{(5)}$

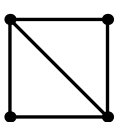

$\mathcal{G}_{4}{ }^{(6)}$

Figure 1: Simple connected graphs on 4 nodes.

Let us denote vertices of graphs $\mathcal{G}_{4}^{(5)}$ and $\mathcal{G}_{4}^{(6)}$ counterclockwise by 1, 2, 3 and 4 starting with the upper right vertex. The characteristic polynomials of the corresponding graph distance matrices

$$
G_{4}^{(5)}=\left[\begin{array}{llll}
0 & 1 & 2 & 2 \\
1 & 0 & 1 & 1 \\
2 & 1 & 0 & 1 \\
2 & 1 & 1 & 0
\end{array}\right] \quad \text { and } G_{4}^{(6)}=\left[\begin{array}{cccc}
0 & 1 & 2 & 1 \\
1 & 0 & 1 & 1 \\
2 & 1 & 0 & 1 \\
1 & 1 & 1 & 0
\end{array}\right]
$$

are

$$
\begin{aligned}
& p_{G_{4}^{(5)}}(\lambda)=(\lambda+1)\left(\lambda^{3}-\lambda^{2}-11 \lambda-7\right), \\
& p_{G_{4}^{(6)}}(\lambda)=(\lambda+1)(\lambda+2)\left(\lambda^{2}-3 \lambda-2\right) .
\end{aligned}
$$

Thus matrices $G_{4}^{(5)}$ and $G_{4}^{(6)}$ have eigenvalues

$$
\sigma_{G_{4}^{(5)}} \doteq\{4.1,-0.7,-1,-2.4\} \quad \text { and } \quad \sigma_{G_{4}^{(6)}}=\left\{\frac{3+\sqrt{17}}{2}, \frac{3-\sqrt{17}}{2},-1,-2\right\} \text {. }
$$

Eigenvalues for $G_{4}^{(5)}$ were calculated numerically. Exact values can be calculated by using Cardano's formula. 
One can easily verify that vectors

$$
\boldsymbol{w}_{G_{4}^{(5)}}=[3 / 7,-1 / 7,2 / 7,2 / 7]^{T} \quad \text { and } \quad \boldsymbol{w}_{G_{4}^{(6)}}=[1 / 2,0,1 / 2,0]^{T}
$$

satisfy the equation $G_{4}^{(i)} \boldsymbol{w}_{G_{4}^{(i)}}=\boldsymbol{e}, i=5,6$. Since $\boldsymbol{w}_{G_{4}^{(i)}}^{T} \boldsymbol{e}>0, i=5,6$, by Theorem 1.1 graphs $\mathcal{G}_{4}^{(5)}$ and $\mathcal{G}_{4}^{(6)}$ are EDM-graphs. Thus there are no NEDM-graphs on 4 nodes.

In the case $n=5$, there are 21 simple connected graphs (see Fig. 2).

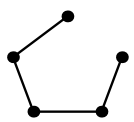

$\mathcal{G}_{5}^{(1)}$

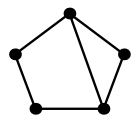

$\mathcal{G}_{5}^{(8)}$

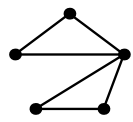

$\mathcal{G}_{5}^{(15)}$

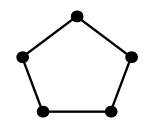

$\mathcal{G}_{5}^{(2)}$

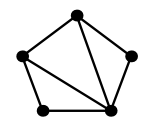

$\mathcal{G}_{5}^{(9)}$

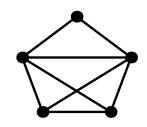

$\mathcal{G}_{5}^{(16)}$

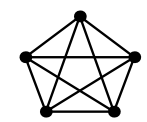

$\mathcal{G}_{5}{ }^{(3)}$

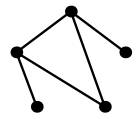

$\mathcal{G}_{5}^{(10)}$

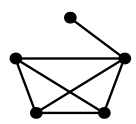

$\mathcal{G}_{5}^{(17)}$

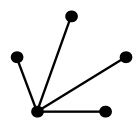

$\mathcal{G}_{5}^{(4)}$

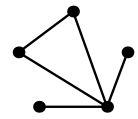

$\mathcal{G}_{5}^{(11)}$

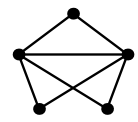

$\mathcal{G}_{5}^{(18)}$

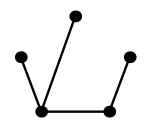

$\mathcal{G}_{5}^{(5)}$

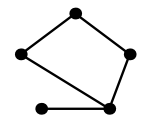

$\mathcal{G}_{5}^{(12)}$

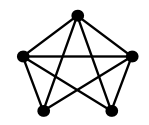

$\mathcal{G}_{5}^{(19)}$

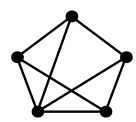

$\mathcal{G}_{5}^{(6)}$

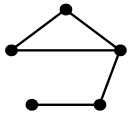

$\mathcal{G}_{5}^{(13)}$

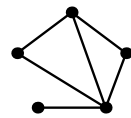

$\mathcal{G}_{5}{ }^{(7)}$

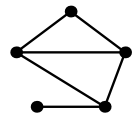

$\mathcal{G}_{5}^{(14)}$

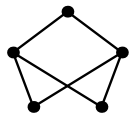

$\mathcal{G}_{5}^{(20)}$

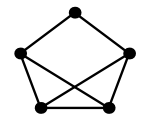

$\mathcal{G}_{5}^{(21)}$

Figure 2: Simple connected graphs on 5 nodes.

Graphs $\mathcal{G}_{5}^{(i)}, i \leq 5$, are the path graph $\mathcal{P}_{5}$, the cycle graph $\mathcal{C}_{5}$, the complete graph $\mathcal{K}_{5}$, the star graph $\mathcal{S}_{5}$ and the tree $\mathcal{T}_{5}$, respectively. Since they are EDM-graphs (see [1]), we only need to analyse graphs $\mathcal{G}_{5}^{(i)}, i=6,7, \ldots, 21$.

A straightforward calculation shows that the graph distance matrix $G_{5}^{(i)}$ of the graph $\mathcal{G}_{5}^{(i)}, i=6,7, \ldots, 19$, has exactly one positive eigenvalue and that there exists $\boldsymbol{w}_{G_{5}^{(i)}} \in \mathbb{R}^{5}$, such that $G_{5}^{(i)} \boldsymbol{w}_{G_{5}^{(i)}}=\boldsymbol{e}$ and $\boldsymbol{w}_{G_{5}^{(i)}}^{T} \boldsymbol{e} \geq 0$. By Theorem 1.1, graphs $\mathcal{G}_{5}^{(6)}, \mathcal{G}_{5}^{(7)}, \ldots, \mathcal{G}_{5}^{(19)}$ are EDM-graphs.

We are left with graphs $\mathcal{G}_{5}^{(20)}$ and $\mathcal{G}_{5}^{(21)}$ (see Fig. 3). The characteristic polynomials of

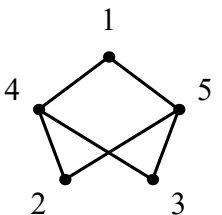

$\mathcal{G}_{5}^{(20)}$

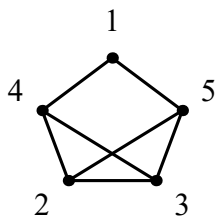

$\mathcal{G}_{5}^{(21)}$

Figure 3: The graphs $\mathcal{G}_{5}^{(20)}$ and $\mathcal{G}_{5}^{(21)}$. 
the corresponding graph distance matrices

$$
G_{5}^{(20)}=\left[\begin{array}{lllll}
0 & 2 & 2 & 1 & 1 \\
2 & 0 & 2 & 1 & 1 \\
2 & 2 & 0 & 1 & 1 \\
1 & 1 & 1 & 0 & 2 \\
1 & 1 & 1 & 2 & 0
\end{array}\right] \quad \text { and } \quad G_{5}^{(21)}=\left[\begin{array}{lllll}
0 & 2 & 2 & 1 & 1 \\
2 & 0 & 1 & 1 & 1 \\
2 & 1 & 0 & 1 & 1 \\
1 & 1 & 1 & 0 & 2 \\
1 & 1 & 1 & 2 & 0
\end{array}\right]
$$

are

$$
\begin{aligned}
& p_{G_{5}^{(20)}}(\lambda)=-(\lambda+2)^{3}\left(\lambda^{2}-6 \lambda+2\right), \\
& p_{G_{5}^{(21)}}(\lambda)=-(\lambda+1)(\lambda+2)\left(\lambda^{3}-3 \lambda^{2}-12 \lambda+2\right) .
\end{aligned}
$$

Thus matrices $G_{5}^{(20)}$ and $G_{5}^{(21)}$ have spectra

$$
\sigma_{G_{5}^{(20)}}=\{3+\sqrt{7}, 3-\sqrt{7},-2,-2,-2\} \quad \text { and } \quad \sigma_{G_{5}^{(21)}} \doteq\{5.2,0.2,-1,-2,-2.4\} .
$$

Exact eigenvalues for $G_{5}^{(21)}$ can be calculated by using Cardano's formula. Here they were calculated numerically. Since matrices $G_{5}^{(20)}$ and $G_{5}^{(21)}$ have two positive eigenvalues, graphs $\mathcal{G}_{5}^{(20)}$ and $\mathcal{G}_{5}^{(21)}$ are NEDM-graphs. These are the smallest NEDM-graphs.

An induced subgraph $\mathcal{H}$ of a graph $\mathcal{G}$ is a subset of the vertices $\mathcal{V}(\mathcal{G})$ together with all edges whose endpoints are both in this subset.

Proposition 2.1. Let $\mathcal{G}$ be a simple connected graph and let $\mathcal{H}$ be its induced subgraph. If $\mathcal{H}$ is a NEDM-graph, the graph $\mathcal{G}$ is a NEDM-graph as well.

Proof. Let $n$ and $m<n$, denote the number of nodes in graphs $\mathcal{G}$ and $\mathcal{H}$, respectively. Let us order vertices of the graph $\mathcal{G}$ in such a way that the first $m$ vertices are the vertices of the graph $\mathcal{H}$. Thus the distance matrix $G$ of the graph $\mathcal{G}$ is of the form

$$
G=\left[\begin{array}{ll}
H & * \\
* & *
\end{array}\right],
$$

where $H$ is the distance matrix of the graph $\mathcal{H}$. Every principal submatrix of an EDM has to be an EDM as well. Thus since $H$ is not an EDM, neither is $G$. Therefore $\mathcal{G}$ is a NEDM-graph.

All NEDM-graphs form a set of forbidden subgraphs of the class of EDM-graphs. Graphs $\mathcal{G}_{5}^{(20)}$ and $\mathcal{G}_{5}^{(21)}$ are the minimal forbidden subgraphs. All minimal forbidden subgraphs on 6 and 7 nodes can be seen in Fig. 4 and Fig. 5.

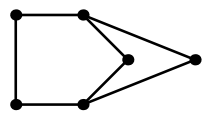

$\mathcal{G}_{6}^{(1)}$

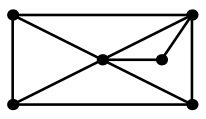

$\mathcal{G}_{6}^{(2)}$

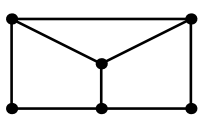

$\mathcal{G}_{6}^{(3)}$

Figure 4: NEDM-graphs for $n=6$. 


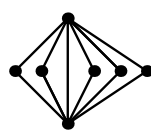

$\mathcal{G}_{7}^{(1)}$

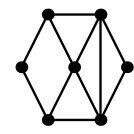

$\mathcal{G}_{7}^{(8)}$

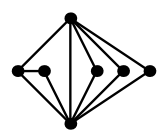

$\mathcal{G}_{7}^{(2)}$

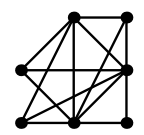

$\mathcal{G}_{7}^{(9)}$

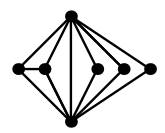

$\mathcal{G}_{7}^{(3)}$

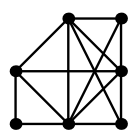

$\mathcal{G}_{7}^{(10)}$

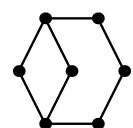

$\mathcal{G}_{7}^{(4)}$

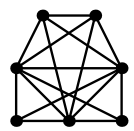

$\mathcal{G}_{7}^{(11)}$

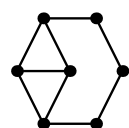

$\mathcal{G}_{7}^{(5)}$

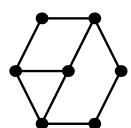

$\mathcal{G}_{7}^{(6)}$

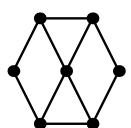

$\mathcal{G}_{7}^{(7)}$

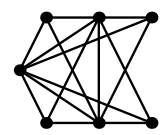

$\mathcal{G}_{7}^{(12)}$

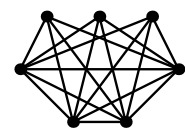

$\mathcal{G}_{7}^{(13)}$

Figure 5: NEDM-graphs for $n=7$.

Let $m(n)$ be the number of NEDM-graphs on $n$ nodes and let $m_{n e w}(n)$ be the number of NEDM-graphs on $n$ nodes for which none of the induced subgraphs is NEDM-graph. We denote the number of non-isomorphic simple connected graphs on $n$ nodes by $g(n)$. Table 1 shows how numbers $m(n)$ and $m_{\text {new }}(n)$ grow with $n$.

The calculations were done in the following way. By using program geng in Nauty ([12]) we generated all simple connected non-isomorphic graphs on $n \leq 8$ nodes. Then we applied Theorem 1.1 to determine whether a graph is an EDM-graph. Computations were done in Mathematica.

\begin{tabular}{|c|c|c|c|}
\hline$n$ & $g(n)$ & $m(n)$ & $m_{n e w}(n)$ \\
\hline 5 & 21 & 2 & 2 \\
\hline 6 & 112 & 27 & 3 \\
\hline 7 & 853 & 341 & 13 \\
\hline 8 & 11117 & 7946 & 48 \\
\hline
\end{tabular}

Table 1: Number of NEDM-graphs compared to the number of all graphs on $n$ nodes.

\section{Bipartite graphs}

A quick observation shows that the graph $\mathcal{G}_{5}^{(20)}$ is bipartite (see Fig. 3).

Let $\mathcal{G}_{\mathcal{U}_{k}, \mathcal{Z}_{n-k}}$ be a simple connected bipartite graph on $n \geq 5$ nodes, whose vertices are divided into two disjoint sets $\mathcal{U}_{k}=\left\{u_{1}, u_{2}, \ldots, u_{k}\right\}, \mathcal{Z}_{n-k}=\left\{u_{k+1}, u_{k+2}, \ldots, u_{n}\right\}$, $k=2,3, \ldots, n-2$, such that every edge connects a vertex in $\mathcal{U}_{k}$ to a vertex in $\mathcal{Z}_{n-k}$ (see Fig. 6). The sets $\mathcal{U}_{k}$ and $\mathcal{Z}_{n-k}$ are called the partition sets.

A graph join $\mathcal{G}_{1}+\mathcal{G}_{2}$ of graphs $\mathcal{G}_{1}$ and $\mathcal{G}_{2}$ with disjoint vertex sets $\mathcal{V}\left(\mathcal{G}_{1}\right), \mathcal{V}\left(\mathcal{G}_{2}\right)$ and edge sets $\mathcal{E}\left(\mathcal{G}_{1}\right), \mathcal{E}\left(\mathcal{G}_{2}\right)$ is the graph with the vertex set $\mathcal{V}\left(\mathcal{G}_{1}\right) \cup \mathcal{V}\left(\mathcal{G}_{2}\right)$ and the edge set $\mathcal{E}\left(\mathcal{G}_{1}\right) \cup \mathcal{E}\left(\mathcal{G}_{2}\right) \cup\left\{(u, v) ; u \in \mathcal{V}\left(\mathcal{G}_{1}\right), v \in \mathcal{V}\left(\mathcal{G}_{2}\right)\right\}$. It is the graph union $\mathcal{G}_{1} \cup \mathcal{G}_{2}$ with all the edges that connect the vertices of the first graph with the vertices of the second graph.

The graph $\mathcal{G}_{\mathcal{U}_{k}, \mathcal{Z}_{n-k}}$ can also be written as the graph join of two empty graphs on $k$ and 
$n-k$ vertices, i.e., $\mathcal{G}_{\mathcal{U}_{k}, \mathcal{Z}_{n-k}}=\mathcal{O}_{k}+\mathcal{O}_{n-k}$. The corresponding graph distance matrix is

$$
G_{k, n-k}=\left[\begin{array}{cc}
2\left(E_{k, k}-I_{k}\right) & E_{k, n-k} \\
E_{n-k, k} & 2\left(E_{n-k, n-k}-I_{n-k}\right)
\end{array}\right] \in \mathbb{R}^{n \times n},
$$

where $E_{p, q} \in \mathbb{R}^{p \times q}$ and $I_{p} \in \mathbb{R}^{p \times p}$ are the matrix of ones and the identity matrix, respectively.

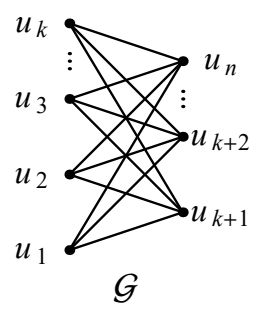

Figure 6: The graph $\mathcal{G}_{\mathcal{U}_{k}, \mathcal{Z}_{n-k}}$.

Theorem 3.1. A simple connected bipartite graph $\mathcal{G}_{\mathcal{U}_{k}, \mathcal{Z}_{n-k}}$ on $n \geq 5$ nodes and with partition sets $\mathcal{U}_{k}$ and $\mathcal{Z}_{n-k}$ is a NEDM-graph.

Proof. Since graphs $\mathcal{G}_{\mathcal{U}_{k}, \mathcal{Z}_{n-k}}$ and $\mathcal{G}_{\mathcal{U}_{n-k}, \mathcal{Z}_{k}}$ are isomorphic, it is enough to see that the theorem holds true for $k=2,3, \ldots,\lfloor n / 2\rfloor$.

Let us analyse the eigenvalues of the graph distance matrix of $\mathcal{G}_{\mathcal{U}_{k}, \mathcal{Z}_{n-k}}$. A simple computation shows that $\boldsymbol{u}_{1, i}=\left[\boldsymbol{e}_{1}^{T}-\boldsymbol{e}_{i}^{T}, \mathbf{0}^{T}\right]^{T}$ solves the equation $G_{k, n-k} \boldsymbol{u}_{1, i}=-2 \boldsymbol{u}_{1, i}$ for all $i=2,3, \ldots, k$, and that $\boldsymbol{u}_{2, j}=\left[\mathbf{0}^{T}, \boldsymbol{e}_{1}^{T}-\boldsymbol{e}_{j}^{T}\right]^{T}$, solves the equation $G_{k, n-k} \boldsymbol{u}_{2, j}=$ $-2 \boldsymbol{u}_{2, j}$ for all $j=2,3, \ldots, n-k$. Therefore $G_{k, n-k}$ has an eigenvalue -2 with multiplicity $n-2$.

Now let us take $\boldsymbol{u}=\left[\alpha \boldsymbol{e}^{T}, \boldsymbol{e}^{T}\right]^{T}$. The relation $G_{k, n-k} \boldsymbol{u}=\lambda \boldsymbol{u}$ yields the system of equations

$$
\begin{aligned}
& 2(k-1) \alpha+n-k=\lambda \alpha, \\
& k \alpha+2(n-k-1)=\lambda,
\end{aligned}
$$

which has solutions

$$
\begin{aligned}
& \alpha_{1,2}=\frac{2 k-n \pm \sqrt{(n-2 k)^{2}+k(n-k)}}{k} \\
& \lambda_{1,2}=n-2 \pm \sqrt{(n-2 k)^{2}+k(n-k)}
\end{aligned}
$$

Relations $n \geq 5$ and $2 \leq k \leq\lfloor n / 2\rfloor$ imply that $\alpha_{1,2}$ and $\lambda_{1,2}$ are well-defined. Since $\lambda_{1}>0$ and

$$
\lambda_{1} \cdot \lambda_{2}=3(k-2)(n-2-k)+2(n-4)>0,
$$

we conclude that $\lambda_{2}>0$. Thus, by Theorem 1.1, the graph $\mathcal{G}_{\mathcal{U}_{k}, \mathcal{Z}_{n-k}}$ is a NEDM-graph.

Remark 3.2. For $k=1$, the graph $\mathcal{G}_{\mathcal{U}_{k}, \mathcal{Z}_{n-k}}$ is the star graph $\mathcal{S}_{n}$, which is an EDM-graph. 


\section{Graph subdivision}

Let $\mathcal{G}$ be a graph. A subdivision of an edge in $\mathcal{G}$ is a substitution of the edge by a path. For example, an edge of the cycle $\mathcal{C}_{n}$ can be subdivided into three edges, resulting in the cycle graph $\mathcal{C}_{n+2}$.

Recall the NEDM-graph $\mathcal{G}_{5}^{(20)}$. It contains a 4-cycle $c$ connecting nodes $2,3,4$ and 5 (see Fig. 7). We can construct larger NEDM-graphs by performing a subdivision of the cycle $c$. Let $\mathcal{G}_{5, n}^{(20)}$ be a graph on $n$ nodes, obtained by subdividing the cycle $c$ in the graph $\mathcal{G}_{5}^{(20)}$ as seen in Fig. 7. Such graphs are $\mathcal{G}_{5,6}^{(20)}=\mathcal{G}_{6}^{(1)}$ and $\mathcal{G}_{5,7}^{(20)}=\mathcal{G}_{7}^{(4)}$ (see Fig. 4 and Fig. 5).
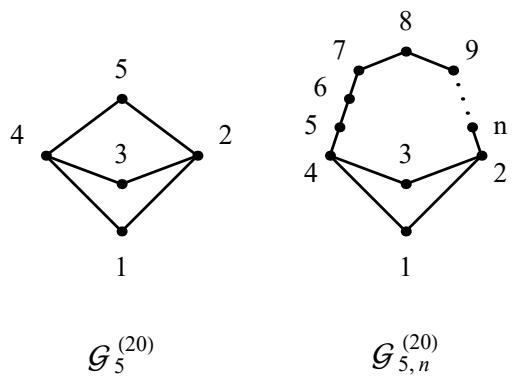

Figure 7: Construction of graphs $\mathcal{G}_{5, n}^{(20)}$.

Let $\boldsymbol{e}_{i}$ denote the standard basis and let $C_{n}$ be the graph distance matrix of the cycle graph $\mathcal{C}_{n}$ (see [9]). The matrix $C_{n}$ is a circulant matrix (see [3]), generated by its first row:

$$
\begin{array}{r}
0,1, \ldots, \frac{n-1}{2}, \frac{n-1}{2}, \frac{n-3}{2}, \ldots, 1, \quad n \text { odd } \\
0,1, \ldots, \frac{n-2}{2}, \frac{n}{2}, \frac{n-2}{2}, \ldots, 1, \quad n \text { even. }
\end{array}
$$

We will use the notation $C_{n}^{(i, j)}$ for the $(i, j)$-th element of the matrix $C_{n}$. The structure of the matrix $C_{n}$ implies

$$
C_{n}^{(1,2)}=C_{n}^{(2,3)}=1, \quad C_{n}^{(1,3)}=2, \quad n \geq 4,
$$

and

$$
C_{n}^{(\ell,\lfloor(n+4) / 2\rfloor)}=\left\{\begin{array}{ll}
\lfloor(n-1) / 2\rfloor, & \ell=1, \\
\lfloor n / 2\rfloor, & \ell=2, \\
\lfloor(n-2) / 2\rfloor, & \ell=3,
\end{array} \quad n \geq 3 .\right.
$$

Theorem 4.1. Graphs $\mathcal{G}_{5, n}^{(20)}, n \geq 5$, are NEDM-graphs.

Proof. The graph distance matrix of the graph $\mathcal{G}_{5, n}^{(20)}, n \geq 5$, is

$$
G_{5, n}^{(20)}=\left[\begin{array}{cc}
0 & \boldsymbol{e}_{2}^{T}\left(C_{n-1}+2 I\right) \\
\left(C_{n-1}+2 I\right) \boldsymbol{e}_{2} & C_{n-1}
\end{array}\right] .
$$

By Theorem 1.1 it is enough to show that there exists $\boldsymbol{x} \in \mathbb{R}^{n}$, such that $\boldsymbol{x}^{T} \boldsymbol{e}=0$ and $\boldsymbol{x}^{T} G_{5, n}^{(20)} \boldsymbol{x}>0$. 
Let us take $\boldsymbol{x}=\left[-\boldsymbol{y}^{T} \boldsymbol{e}, \boldsymbol{y}^{T}\right]^{T}$, with

$$
\boldsymbol{y}= \begin{cases}\frac{n-1}{2}\left(-\boldsymbol{e}_{1}+\boldsymbol{e}_{2}-\boldsymbol{e}_{3}\right)+\boldsymbol{e}_{(n+3) / 2}, & n \text { odd } \\ \frac{n-2}{2}\left(-\boldsymbol{e}_{1}+\frac{n-1}{n}\left(\boldsymbol{e}_{2}-\boldsymbol{e}_{3}\right)\right)+\frac{n-1}{n} \boldsymbol{e}_{(n+2) / 2}, & n \text { even }\end{cases}
$$

We will show that

$$
\boldsymbol{x}^{T} G_{5, n}^{(20)} \boldsymbol{x}=\boldsymbol{y}^{T} C_{n-1} \boldsymbol{y}-2\left(\boldsymbol{y}^{T} \boldsymbol{e}\right)\left(\boldsymbol{e}_{2}^{T} C_{n-1} \boldsymbol{y}+2\left(\boldsymbol{y}^{T} \boldsymbol{e}_{2}\right)\right)>0
$$

From

$$
\boldsymbol{y}^{T} \boldsymbol{e}=\left\{\begin{array}{ll}
\frac{3-n}{2}, & n \text { odd }, \\
\frac{-n^{2}+4 n-2}{2 n}, & n \text { even, }
\end{array} \quad \text { and } \quad \boldsymbol{y}^{T} \boldsymbol{e}_{2}= \begin{cases}\frac{n-1}{2}, & n \text { odd } \\
\frac{(n-2)(n-1)}{2 n}, & n \text { even }\end{cases}\right.
$$

it follows that

$$
\boldsymbol{x}^{T} G_{5, n}^{(20)} \boldsymbol{x}=\boldsymbol{y}^{T} C_{n-1} \boldsymbol{y}+ \begin{cases}(n-3)\left(\boldsymbol{e}_{2}^{T} C_{n-1} \boldsymbol{y}+n-1\right), & n \text { odd } \\ \frac{n^{2}-4 n+2}{n}\left(\boldsymbol{e}_{2}^{T} C_{n-1} \boldsymbol{y}+\frac{(n-2)(n-1)}{n}\right), & n \text { even } .\end{cases}
$$

Firstly, let $n$ be odd. Terms in the relation (4.4) simplify to

$$
\begin{gathered}
\boldsymbol{y}^{T} C_{n-1} \boldsymbol{y}=-\frac{(n-1)^{2}}{2}\left(C_{n-1}^{(1,2)}-C_{n-1}^{(1,3)}+C_{n-1}^{(2,3)}\right)- \\
-(n-1)\left(C_{n-1}^{(1,(n+3) / 2)}-C_{n-1}^{(2,(n+3) / 2)}+C_{n-1}^{(3,(n+3) / 2)}\right), \\
\boldsymbol{e}_{2}^{T} C_{n-1} \boldsymbol{y}=C_{n-1}^{(2,(n+3) / 2)}-\frac{n-1}{2}\left(C_{n-1}^{(1,2)}+C_{n-1}^{(2,3)}\right) .
\end{gathered}
$$

By (4.1) and (4.2),

$$
\boldsymbol{y}^{T} C_{n-1} \boldsymbol{y}=-\frac{(n-1)(n-5)}{2}, \quad \boldsymbol{e}_{2}^{T} C_{n-1} \boldsymbol{y}=-\frac{n-1}{2},
$$

and

$$
\boldsymbol{x}^{T} G_{5, n}^{(20)} \boldsymbol{x}=n-1,
$$

which satisfies the requirement (4.3) for all $n \geq 5$.

When $n$ is even, the terms in the relation (4.4) simplify to

$$
\begin{aligned}
\boldsymbol{y}^{T} C_{n-1} \boldsymbol{y}=- & \frac{(n-2)(n-1)}{2 n^{2}}\left((n-2)\left(n C_{n-1}^{(1,2)}-n C_{n-1}^{(1,3)}+(n-1) C_{n-1}^{(2,3)}\right)+\right. \\
& \left.+2 n C_{n-1}^{(1,(n+2) / 2)}-2(n-1)\left(C_{n-1}^{(2,(n+2) / 2)}-C_{n-1}^{(3,(n+2) / 2)}\right)\right), \\
\boldsymbol{e}_{2}^{T} C_{n-1} \boldsymbol{y}= & \frac{n-1}{n} C_{n-1}^{(2,(n+2) / 2)}-\frac{n-2}{2}\left(C_{n-1}^{(1,2)}+\frac{n-1}{n} C_{n-1}^{(2,3)}\right) .
\end{aligned}
$$

By (4.1) and (4.2),

$$
\boldsymbol{y}^{T} C_{n-1} \boldsymbol{y}=-\frac{(n-1)^{2}(n-2)(n-4)}{2 n^{2}}, \quad \boldsymbol{e}_{2}^{T} C_{n-1} \boldsymbol{y}=-\frac{n-2}{2}
$$


and

$$
\boldsymbol{x}^{T} G_{5, n}^{(20)} \boldsymbol{x}=\frac{n-2}{2 n},
$$

which satisfies the requirement (4.3) for all $n \geq 5$.

Similarly, we can subdivide cycles of the graph $\mathcal{G}_{5}^{(21)}$ and produce NEDM-graphs (see Fig. 8). The graph $\mathcal{G}_{5}^{(21)}$ contains a 3 -cycle $c$ connecting nodes 3,4 and 5 . Let $\mathcal{G}_{5, n}^{(21)}$ be a graph on $n$ nodes, obtained by subdividing the cycle $c$ in the graph $\mathcal{G}_{5}^{(21)}$ as seen in Fig. 8 . Such graphs are $\mathcal{G}_{5,5}^{(21)}=\mathcal{G}_{5}^{(21)}, \mathcal{G}_{5,6}^{(21)}=\mathcal{G}_{6}^{(3)}$ and $\mathcal{G}_{5,7}^{(21)}=\mathcal{G}_{7}^{(6)}$ (see Fig. 4 and Fig. 5).

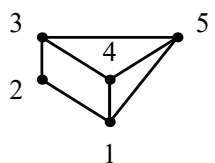

$\mathcal{G}_{5}^{(21)}$

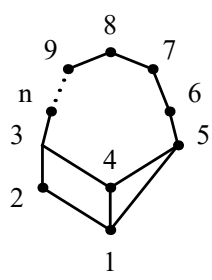

$\mathcal{G}_{5, n}^{(21)}$

Figure 8: Graphs $\mathcal{G}_{5, n}^{(21)}$.

Theorem 4.2. Graphs $\mathcal{G}_{5, n}^{(21)}, n \geq 5$, are NEDM-graphs.

Proof. The graph distance matrix of the graph $\mathcal{G}_{5, n}^{(21)}, n \geq 5$, is

$$
G_{5, n}^{(21)}=\left[\begin{array}{ccc}
0 & 1 & \boldsymbol{u}^{T} \\
1 & 0 & \boldsymbol{v}^{T} \\
\boldsymbol{u} & \boldsymbol{v} & C_{n-2}
\end{array}\right]
$$

where

$$
\boldsymbol{u}=\left(C_{n-2}+I\right) \boldsymbol{e}_{2}+\boldsymbol{e}-\boldsymbol{y}-\frac{1+(-1)^{n}}{2} \boldsymbol{e}_{(n+2) / 2}, \quad \boldsymbol{v}=\left(C_{n-2}+I\right) \boldsymbol{e}_{2}+\boldsymbol{y}
$$

and $\boldsymbol{y}=\sum_{k=2}^{\lfloor(n+1) / 2\rfloor} \boldsymbol{e}_{k}$. Analogous to the proof of Theorem 4.1, we can show that for $\boldsymbol{x}=\left[\alpha,-\alpha, \boldsymbol{z}^{T}\right]^{T}$, where

$$
\alpha=\left\{\begin{array}{ll}
\frac{n}{2}, & n \text { odd }, \\
\frac{n-3}{2}, & n \text { even, }
\end{array} \quad \text { and } \boldsymbol{z}= \begin{cases}\frac{n-1}{2} \boldsymbol{e}_{1}-\frac{n-3}{2} \boldsymbol{e}_{2}-\boldsymbol{e}_{(n+1) / 2}, & n \text { odd } \\
\frac{n-2}{2} \boldsymbol{e}_{1}-\frac{n-4}{2} \boldsymbol{e}_{2}-\boldsymbol{e}_{(n+2) / 2}, & n \text { even }\end{cases}\right.
$$

the expression $\boldsymbol{x}^{T} G_{5, n}^{(21)} \boldsymbol{x}=\boldsymbol{z}^{T} C_{n-2} \boldsymbol{z}+2 \alpha\left(\boldsymbol{u}^{T} \boldsymbol{z}-\boldsymbol{v}^{T} \boldsymbol{z}-\alpha\right)$ is positive.

Relations

$$
\begin{aligned}
C_{n-2}^{(1,2)} & =1, & C_{n-2}^{(1,(n+1) / 2)} & =C_{n-2}^{(2,(n+1) / 2)}=\frac{n-3}{2}, \\
C_{n-2}^{(1,(n+2) / 2)} & =\frac{n-4}{2}, & C_{n-2}^{(2,(n+2) / 2)} & =\frac{n-2}{2},
\end{aligned}
$$


imply

$$
\boldsymbol{u}^{T} \boldsymbol{z}=\left\{\begin{array}{ll}
2, & n \text { odd, } \\
1, & n \text { even },
\end{array} \quad \boldsymbol{v}^{T} \boldsymbol{z}= \begin{cases}3-n, & n \text { odd } \\
4-n, & n \text { even }\end{cases}\right.
$$

and

$$
\boldsymbol{z}^{T} C_{n-2} \boldsymbol{z}= \begin{cases}-\frac{(n-3)(n+1)}{2}, & n \text { odd } \\ -\frac{(n-2)(n-4)}{2}, & n \text { even }\end{cases}
$$

which yields

$$
\boldsymbol{x}^{T} G_{5, n}^{(21)} \boldsymbol{x}= \begin{cases}\frac{3}{2}, & n \text { odd } \\ \frac{1}{2}, & n \text { even } .\end{cases}
$$

Thus by Theorem 1.1, the matrix $G_{5, n}^{(21)}$ is a NEDM.

\section{Systems with no solution}

When verifying whether a graph $\mathcal{G}$ with the corresponding graph distance matrix $G$ is an EDM-graph, by Theorem 1.1 one can check if there exists a solution of the equation $G \boldsymbol{w}=\boldsymbol{e}$, such that $\boldsymbol{w}^{T} \boldsymbol{e} \geq 0$. For $n \geq 7$ there exist graphs, for which the equation $G \boldsymbol{w}=\boldsymbol{e}$ has no solution.

Let $\mathcal{G}_{k, n-k}$ be the graph join of a complete graph $\mathcal{K}_{k}$ and an empty graph $\mathcal{O}_{n-k}, n \geq 7$, $k=2,3, \ldots, n-3$, i.e.,

$$
\mathcal{G}_{k, n-k}=\mathcal{K}_{k}+\mathcal{O}_{n-k} .
$$

The graph $\mathcal{K}_{k}$ contains vertices $1,2, \ldots, k$ and the graph $\mathcal{O}_{n-k}$ contains vertices $k+1, k+$ $2, \ldots, n$. Thus the corresponding graph distance matrix is

$$
G_{k, n-k}=\left[\begin{array}{cc}
E_{k, k}-I_{k} & E_{k, n-k} \\
E_{n-k, k} & 2\left(E_{n-k, n-k}-I_{n-k}\right)
\end{array}\right] .
$$

For $n=7$ and $k=3$ the equation $G_{3,4} \boldsymbol{w}=\boldsymbol{e}$ has no solution since the ranks of the matrix $G_{3,4}$ and its augmented matrix $\left[G_{3,4} \mid e\right]$ are different, $\operatorname{rank}\left(G_{3,4}\right)=6$ and $\operatorname{rank}\left(\left[G_{3,4} \mid \boldsymbol{e}\right]\right)=7$. The same holds true if $n=7$ and $k=4$. Thus by Theorem 1.1 matrices $G_{3,4}$ and $G_{4,3}$ are not EDMs. On the other hand, for $n=8$ the equation $G_{k, 8-k} \boldsymbol{w}=\boldsymbol{e}$ has a solution for all $k \in\{3,4,5\}$. In general, the matrix $G_{k, n-k}$ is a NEDM.

Theorem 5.1. The graph $\mathcal{K}_{k}+\mathcal{O}_{n-k}, n \geq 7, k=2,3, \ldots, n-3$, is a NEDM-graph.

Proof. Let $G_{k, n-k}$ be the graph distance matrix of the graph $\mathcal{K}_{k}+\mathcal{O}_{n-k}, n \geq 7, k=$ $2,3, \ldots, n-3$. For $k=2$ we take

$$
\boldsymbol{w}=\frac{1}{2}[4-n, 4-n, 1,1, \ldots, 1]^{T} .
$$

We can verify that $G_{2, n-2} \boldsymbol{w}=\boldsymbol{e}$ and $\boldsymbol{w}^{T} \boldsymbol{e}=(6-n) / 2<0$. Thus by Theorem 1.1 the matrix $G_{2, n-2}$ is a NEDM.

Now let $k=3,4, \ldots, n-3$. For $n=7$ the proof has already been done above. For $n \geq 8$ let $\boldsymbol{u}=\left[\alpha \boldsymbol{e}^{T}, \boldsymbol{e}^{T}\right]^{T}$, where vectors $\boldsymbol{e}$ are of sizes $k$ and $n-k$, respectively. The relation $G_{k, n-k} \boldsymbol{u}=\lambda \boldsymbol{u}$ yields the system of equations

$$
\begin{aligned}
\alpha(k-1)+n-k & =\lambda \alpha, \\
\alpha k+2(n-k-1) & =\lambda,
\end{aligned}
$$


with solutions

$$
\begin{aligned}
& \alpha_{1,2}=\frac{1}{2 k}\left(3 k-2 n+1 \pm \sqrt{4(n-k)(n-k-1)+(k+1)^{2}}\right), \\
& \lambda_{1,2}=\frac{1}{2}\left(2 n-k-3 \pm \sqrt{4(n-k)(n-k-1)+(k+1)^{2}}\right) .
\end{aligned}
$$

Relations $n \geq 8$ and $3 \leq k \leq n-3$ imply that $\alpha_{1,2}$ and $\lambda_{1,2}$ are well-defined. Since $\lambda_{1}>0$ and

$$
\lambda_{1} \cdot \lambda_{2}=(n-3-k)(k-3)+n-7>0,
$$

we conclude that $\lambda_{2}>0$. Thus, by Theorem 1.1, graph $\mathcal{K}_{k}+\mathcal{O}_{n-k}$ is a NEDM-graph.

Remark 5.2. For $k=1$ and $k=n-1$, the graphs $\mathcal{K}_{k}+\mathcal{O}_{n-k}$ are the star graph $\mathcal{S}_{n}$ and the complete graph $\mathcal{K}_{n}$, respectively, which are EDM-graphs.

Remark 5.3. For $k=n-2$, the graph $\mathcal{K}_{n-2}+\mathcal{O}_{2}$ is an EDM-graph. The graph distance matrix $G_{n-2,2}$ has eigenpairs

$$
\left(-2,\left[\mathbf{0}^{T}, \boldsymbol{e}_{1}^{T}-\boldsymbol{e}_{2}^{T}\right]^{T}\right), \quad\left(-1,\left[\boldsymbol{e}_{1}^{T}-\boldsymbol{e}_{i}^{T}, \mathbf{0}^{T}\right]^{T}\right), \quad i=2,3, \ldots, n-2,
$$

and

$$
\left(\lambda_{1,2},\left[\alpha_{1,2} e^{T}, e^{T}\right]^{T}\right)
$$

with

$$
\alpha_{1,2}=\frac{n-5 \pm \sqrt{n^{2}-2 n+9}}{2(n-2)} \quad \text { and } \quad \lambda_{1,2}=\frac{n-1 \pm \sqrt{n^{2}-2 n+9}}{2} .
$$

The eigenvalue $\lambda_{1}$ is obviously positive. From $\lambda_{1} \cdot \lambda_{2}=-2$ it follows that $\lambda_{2}<0$. One can easily verify that $\boldsymbol{w}=(1 / 2)\left[\mathbf{0}^{T}, \boldsymbol{e}^{T}\right]^{T}$ solves the equation $G_{n-2,2} \boldsymbol{w}=\boldsymbol{e}$. Since $\boldsymbol{w}^{T} \boldsymbol{e}=1$, Theorem 1.1 implies that $G_{n-2,2}$ is EDM.

\section{Conclusion}

In Section 4 we studied subdivisions of graphs. Not all graph subdivisions result in NEDMgraphs. Consider subdividing graph $\mathcal{G}_{5}^{(20)}$ as in Fig. 9 and denoting it by $\mathcal{H}$. The corresponding graph distance matrix

$$
H=\left[\begin{array}{lllllll}
0 & 1 & 2 & 2 & 3 & 2 & 1 \\
1 & 0 & 1 & 2 & 2 & 1 & 2 \\
2 & 1 & 0 & 1 & 2 & 2 & 2 \\
2 & 2 & 1 & 0 & 1 & 2 & 1 \\
3 & 2 & 2 & 1 & 0 & 1 & 2 \\
2 & 1 & 2 & 2 & 1 & 0 & 3 \\
1 & 2 & 2 & 1 & 2 & 3 & 0
\end{array}\right]
$$

has eigenvalues $\sigma_{H} \doteq\{10.4,0,-0.2,-0.6,-2.2,-3.4,-4\}$, which were calculated numerically. Exact eigenvalues could be obtained using Cardano's formula. One can easily verify that vector $\boldsymbol{w}_{H}=[1 / 2,-1 / 2,1 / 2,-1 / 2,1 / 2,0,0]^{T}$ solves the equation $H \boldsymbol{w}_{H}=\boldsymbol{e}$. Since $\boldsymbol{w}_{H}^{T} \boldsymbol{e}=1 / 2$, by Theorem 1.1 the graph $\mathcal{H}$ is an EDM-graph. 


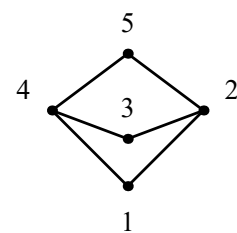

$\mathcal{G}_{5}^{(20)}$

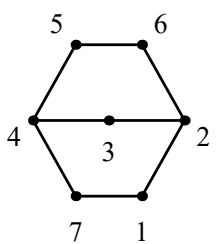

$\mathcal{H}$

Figure 9: A subdivision of the graph $\mathcal{G}_{5}^{(20)}$.

\section{References}

[1] R. Balaji and R. B. Bapat, Block distance matrices, Electron. J. Linear Algebra 16 (2007), 435-443.

[2] J. Dattorro, Convex Optimization and Euclidean Distance Geometry, Meboo, 2008.

[3] G. H. Golub and C.F. van Loan, Matrix computations, Johns Hopkins Studies in the Mathematical Sciences, third ed., Johns Hopkins University Press, 1996.

[4] J. C. Gower, Euclidean distance geometry, Math. Sci. 7 (1982), 1-14.

[5] T. L. Hayden, R. Reams and J. Wells, Methods for constructing distance matrices and the inverse eigenvalue problem, Linear Algebra Appl. 295 (1999), 97-112.

[6] G. Jaklič, J. Modic, On properties of cell matrices, Appl. Math. Comput. 216 (2010), 20162023.

[7] G. Jaklič, J. Modic, A note on "Methods for constructing distance matrices and the inverse eigenvalue problem”, Linear Algebra Appl. 437 (2012), 2781-2792.

[8] G. Jaklič and J. Modic, Inverse eigenvalue problem for Euclidean distance matrices of size 3, Bull. Aust. Math. Soc. 87 (2013), 82-93.

[9] G. Jaklič and J. Modic, On Euclidean distance matrices of graphs, Electron. J. Linear Algebra 26 (2013), 574-589.

[10] G. Jaklič, J. Modic, Euclidean graph distance matrices of generalizations of the star graph, Appl. Math. Comput. 230 (2014), 650-663.

[11] G. Jaklič, J. Modic, Cartesian products of EDM-graphs, submitted.

[12] B. D. McKay, Practical Graph Isomorphism, Congr. Numer. 30 (1981), 45-87.

[13] I. J. Schoenberg, Metric spaces and positive definite functions, Trans. Amer. Math. Soc. 44 (1938), 522-536.

[14] G. Young, A. Householder, Discussion of a set of points in terms of their mutual distances, Psychometrika 3 (1938), 19-22. 